\title{
Mass-Like Extreme Wall Thickening of the Entire Common Bile Duct in a Case of Portal Cavernoma Cholangiopathy
}

\author{
Veeraraghavan Gunasekaran ${ }^{10}$ Supraja Laguduva Mohan ${ }^{1}$ \\ Sunitha Vellathussery Chakkalakkoombil ${ }^{10}$ Kuppusamy Senthamizhselvan ${ }^{2}$

\footnotetext{
${ }^{1}$ Department of Radiodiagnosis, Jawaharlal Institute of Postgraduate Medical Education and Research (JIPMER), Puducherry, India

2 Department of Medical Gastroenterology, Jawaharlal Institute of Postgraduate Medical Education and Research (JIPMER), Puducherry, India
}

\begin{abstract}
Address for correspondence Veeraraghavan Gunasekaran, MD, Department of Radiodiagnosis, Jawaharlal Institute of Postgraduate Medical Education and Research (JIPMER), Puducherry 605006, India (e-mail: raghavan71287@gmail.com).
\end{abstract}

J Gastrointestinal Abdominal Radiol ISGAR 2022;5:63-69.

\begin{abstract}
Keywords

- common bile duct

- magnetic resonance imaging

- portal vein

Portal cavernoma cholangiopathy (PCC) refers to the cholangiographic abnormalities that occur in patients with portal cavernoma. These abnormalities may be either due to chronic portal vein thrombosis or extrahepatic portal vein occlusion. These abnormalities occur due to enlargement of the bridging tortuous paracholedochal, epicholedochal, and cholecystic veins exerting pressure on the bile ducts. Ischemic changes can also occur in the bile duct due to portal vein thrombosis, which affects the microvascular circulation or associated hepatic artery thrombosis. These may be either reversible with shunt procedures such as transjugular intrahepatic portosystemic shunt (TIPS) or irreversible in the advanced stage, leading to recurrent episodes of biliary pain, cholangitis, and cholestasis. Occasionally it may present as mass-like diffuse common bile duct (CBD) wall thickening, which may be confused with mimickers like primary CBD lymphoma, immunoglobulin G4-related sclerosing cholangitis, and even cholangiocarcinoma on imaging. Thus, we need to be aware of the mass-forming PCC imaging findings to avoid unnecessary invasive procedures like biopsy or surgical intervention. Here, we present a case of PCC, which presented as mass-like diffuse CBD wall thickening with patent lumen on ultrasound that led to further workup with contrast-enhanced computed tomography and magnetic resonance imaging. The wall thickening showed persistent delayed enhancement, no significant diffusion restriction, and there was also associated superior mesenteric vein thrombosis with multiple mesenteric collaterals. A positron emission tomography-CT scan also ruled out malignant disease as there was no uptake. Finally, a diagnosis of mass-forming PCC was made by combining imaging features and excluding other causes.
\end{abstract}

published online

November 2, 2021
DOI https://doi.org/

10.1055/s-0041-1735216. ISSN 2581-9933.
(C) 2021. Indian Society of Gastrointestinal and Abdominal Radiology. All rights reserved.

This is an open access article published by Thieme under the terms of the Creative Commons Attribution-NonDerivative-NonCommercial-License, permitting copying and reproduction so long as the original work is given appropriate credit. Contents may not be used for commercial purposes, or adapted, remixed, transformed or built upon. (https://creativecommons.org/ licenses/by-nc-nd/4.0/)

Thieme Medical and Scientific Publishers Pvt. Ltd., A-12, 2nd Floor, Sector 2, Noida-201301 UP, India 


\section{Introduction}

Abnormalities of the biliary tract due to portal cavernoma of chronic portal vein thrombosis were first described as a separate entity by Fraser and Brown in $1944 .^{1}$ Since then, it has been described under various terms, including "portal biliopathy," "portal cholangiopathy," "portal hypertensive biliopathy," "pseudosclerosing cholangitis," and 'pseudocholangiocarcinoma." The current preferred terminology is "portal cavernoma cholangiopathy" (PCC) which was agreed upon as the consensus nomenclature by the Working Party of the Indian National Association for Study of the Liver since it implied the presence of a portal cavernoma resulting in abnormalities of the biliary tree including extra- and intrahepatic bile ductular system, gallbladder, and cystic duct. ${ }^{2}$ The great mimickers of mass-forming PCC include primary common bile duct (CBD) lymphoma, immunoglobulin (Ig) G4-related sclerosing cholangitis, and cholangiocarcinoma. Thus, clinicians must be aware of the clinical presentation and the imaging findings to differentiate it from these conditions, therefore can avoid unnecessary invasive procedures and surgical intervention.

\section{Case Report}

A 35-year-old female patient presented with abdominal pain over the past 3 months, which was localized to the left hypochondrium and the pelvis. Her past medical history was unremarkable. Abdomen examination was not contributory. Blood investigations showed hemoglobin $14.3 \mathrm{~g} / \mathrm{dL}$, white blood cell count $14.79^{*} 10^{3} / \mu \mathrm{L}$, platelet count $455^{*} 10^{3} / \mu \mathrm{L}$, normocytic normochromic red blood cells, total bilirubin $1.04 \mathrm{mg} / \mathrm{dL}$, direct bilirubin $0.24 \mathrm{mg} / \mathrm{dL}$, total protein $7.6 \mathrm{~g} / \mathrm{dL}$, albumin $3.9 \mathrm{~g} / \mathrm{dL}$, aspartate transaminase $29 \mathrm{IU} / \mathrm{L}$, alanine transaminase $46 \mathrm{IU} / \mathrm{L}$, alkaline phosphatase $132 \mathrm{IU} / \mathrm{L}$, and gamma-glutamyl transferase $60 \mathrm{IU} / \mathrm{L}$. An ultrasound examination of the abdomen was done with curvilinear and linear probes using the Siemens Acuson S3000 ultrasound machine (Erlangen, Germany). It showed diffuse wall thickening of the CBD in the form of an isoechoic lesion extending between the porta hepatis and head of the pancreas (-Fig. 1A, B). The maximum wall thickness was $9 \mathrm{~mm}$, and despite such wall thickening, the lumen of the CBD was patent, measuring approximately $5 \mathrm{~mm}$. The liver was normal in morphology and echotexture. The lesion showed rich internal vascularity (-Fig. 2). There was also mild splenomegaly, with the spleen measuring around $13.2 \mathrm{~cm}$ with rounded margins. Further color Doppler of the abdominal vessels revealed superior mesenteric vein thrombosis (-Fig. 3) with multiple mesenteric and perisplenic collaterals. The portal and splenic veins could not be separately delineated. Multiple calcified fibroids were also noted in the uterus.

The triple-phase contrast-enhanced computed tomography (CECT) was done using Siemens SOMATOM Definition Edge 128 slice CT machine (Erlangen, Germany) which revealed an enhancing mass-like wall thickening of the CBD with a maximum wall thickness of $9.6 \mathrm{~mm}$ with homogeneous persistent delayed enhancement ( - Fig. 4A-D). Mild
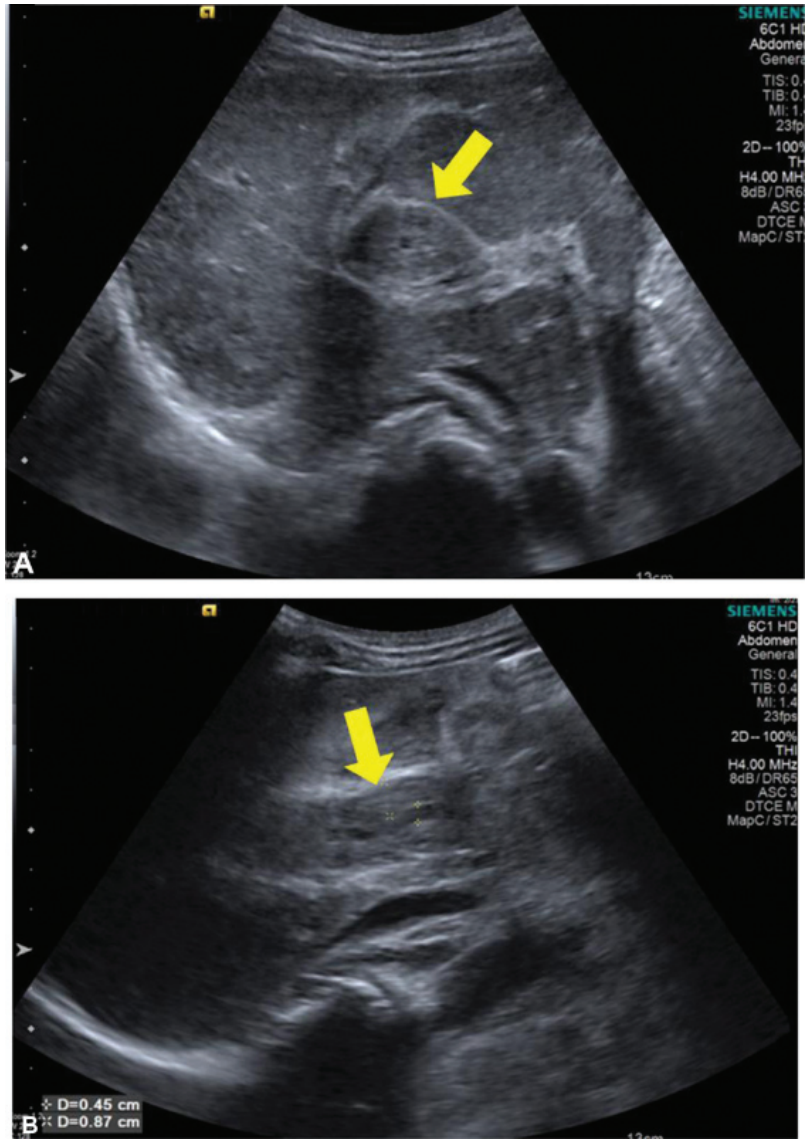

Fig. 1 (A, B) Transabdominal gray scale ultrasonography images show diffuse wall thickening of the common bile duct, in the form of an isoechoic lesion (arrows) extending between the porta hepatis and the head of pancreas.

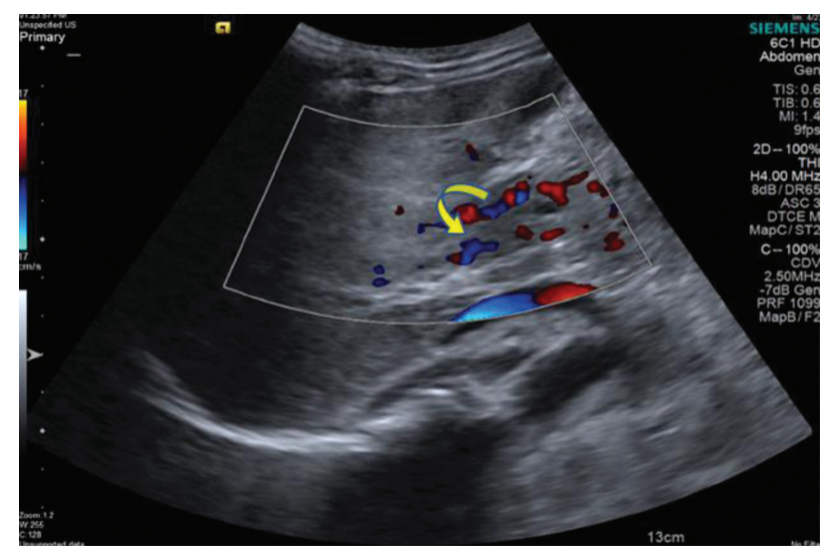

Fig. 2 Transabdominal color Doppler ultrasound images show rich internal vascularity (curved arrows) of the common bile duct wall thickening.

central intrahepatic biliary radicle dilatation was noted. Portal vein and splenic vein were not separately visualized and were replaced by collaterals ( - Fig. 5A, B). Superior mesenteric vein thrombosis was also noted (-Fig. 6). Besides, there was also diffuse retroperitoneal fat stranding in the para-aortic region. We considered differentials like primary CBD lymphoma, IgG4-related sclerosing cholangitis, 


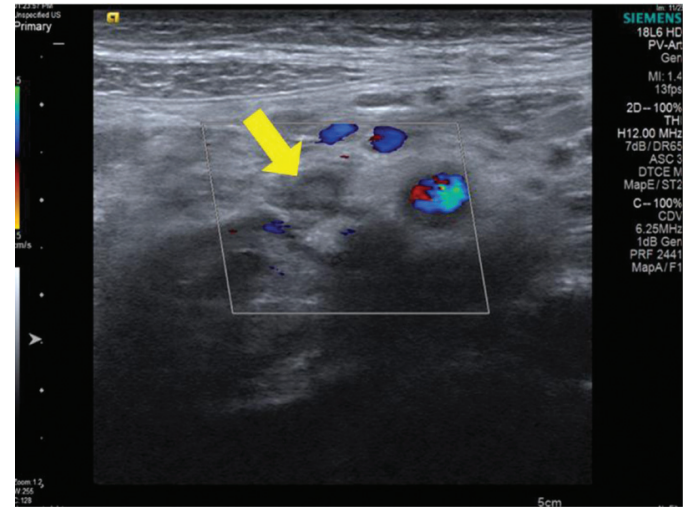

Fig. 3 Transabdominal color Doppler ultrasound (US) images show partial color filling defect (arrow) involving the superior mesenteric vein suggestive of thrombosis.

cholangiocarcinoma, and PCC. Serum IgG4 levels were also found to be normal $(0.496 \mathrm{IU} / \mathrm{mL})$. Antinuclear antibody (ANA) was negative, carbohydrate antigen 19-9 level was $3.5 \mathrm{U} / \mathrm{mL}$ (normal range: $0-37 \mathrm{U} / \mathrm{mL}$ ). The workup for hypercoagulable states namely JAK-2 mutation, factor $\mathrm{V}$ Leiden mutation, protein $C$ and $S$ levels, and antithrombin III levels were unremarkable.
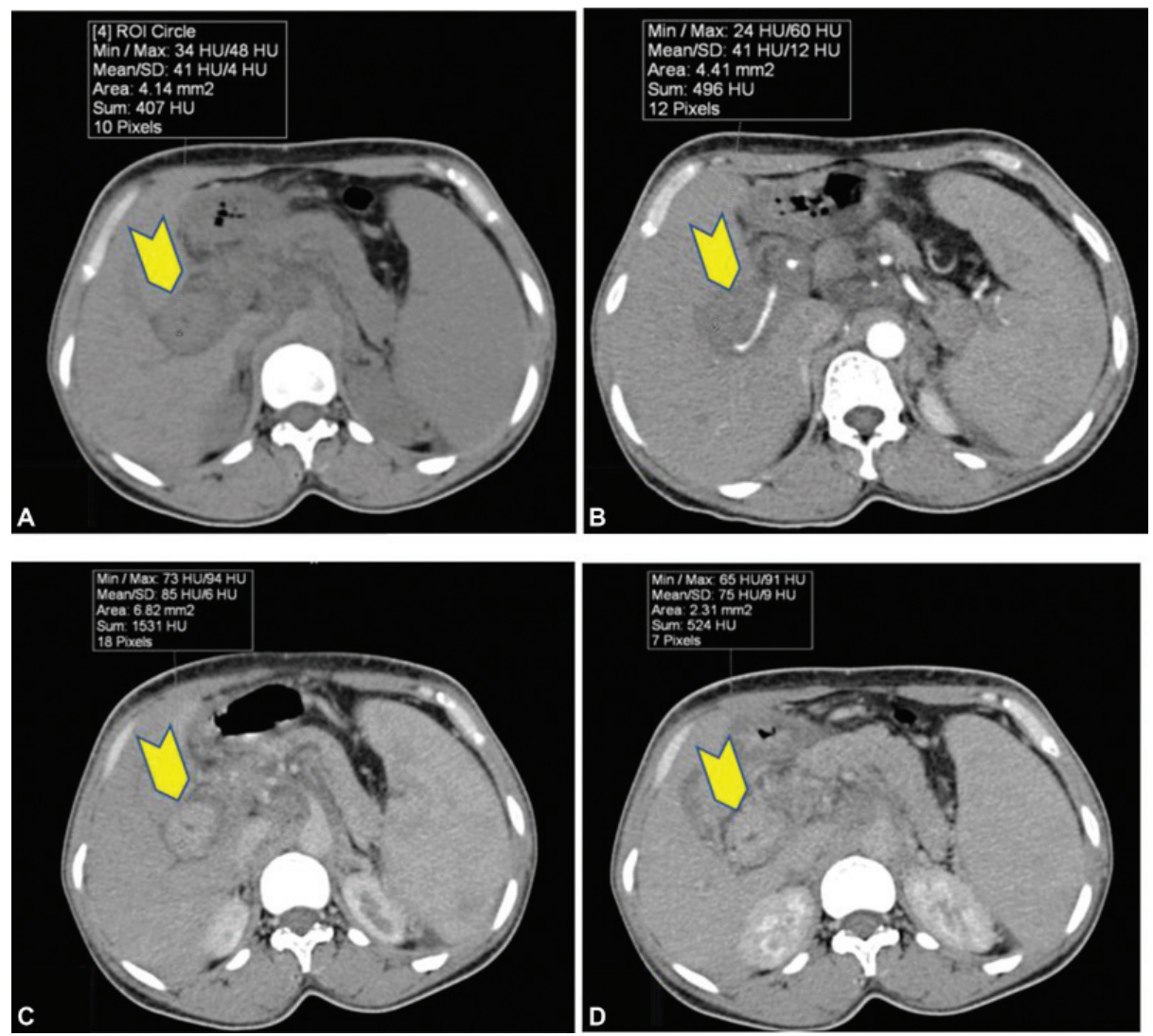

Fig. 4 Axial plain (A) and triphasic contrast computed tomography abdomen images show no obvious enhancement in arterial phase (B) with homogeneous persistent enhancement of the common bile duct wall thickening on portal (C) and delayed (D) phases (chevron).
Magnetic resonance imaging (MRI) of the abdomen along with magnetic resonance cholangiopancreatography (MRCP) was performed using Siemens Avanto 1.5 Tesla (Erlangen, Germany) MRI machine for a detailed evaluation of the CBD pathology. The CBD wall thickening was iso- to hypointense T1W images and hyperintense on T2/STIR (short tau inversion recovery) images with persistent enhancement in the delayed dynamic contrast study phase. There was no obvious diffusion restriction (-Fig. 7). T2 hyperintense signals in the retroperitoneum correspond to the fat stranding seen on CT. The pancreas appeared normal. On MRCP, there was no obvious luminal narrowing of the $\mathrm{CBD}$. A positron emission tomography (PET)-CT was done subsequently which showed no obvious metabolic activity in the concentric wall thickening of the CBD (-Fig. 8). The absence of PET-CT uptake and diffusion restriction ruled out malignant diseases like lymphoma and cholangiocarcinoma. Though there was retroperitoneal fat stranding, there was no retroperitoneal fibrosis as such, there was no luminal narrowing of CBD, and the pancreas was also normal. These features, along with the presence of superior mesenteric vein, chronic portal, and splenic vein thrombosis with multiple collaterals, led to the final diagnosis of a mass-forming PCC. She was treated with anticoagulants and she is currently doing well on regular follow-up. 


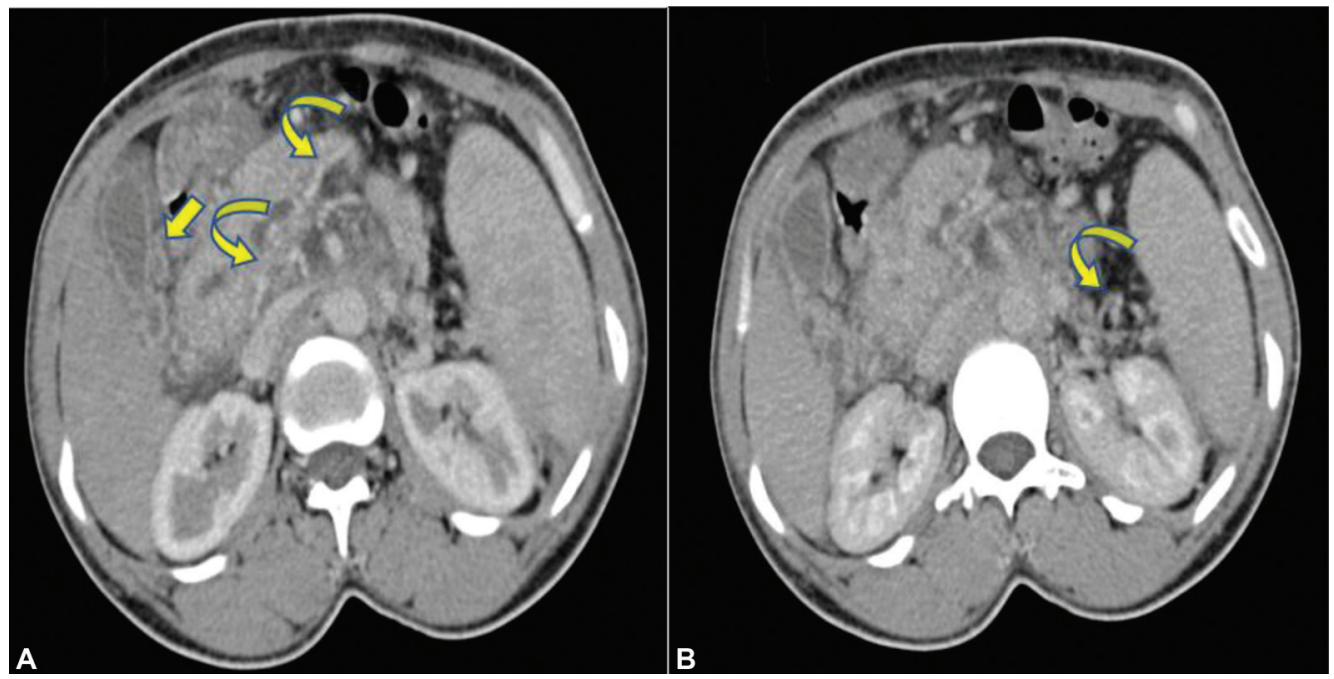

Fig. 5 (A, B) Axial portal phase contrast computed tomography images show collaterals replacing the portal and splenic veins (curved arrows). Few pericholecystic collaterals (arrow) are also noted.

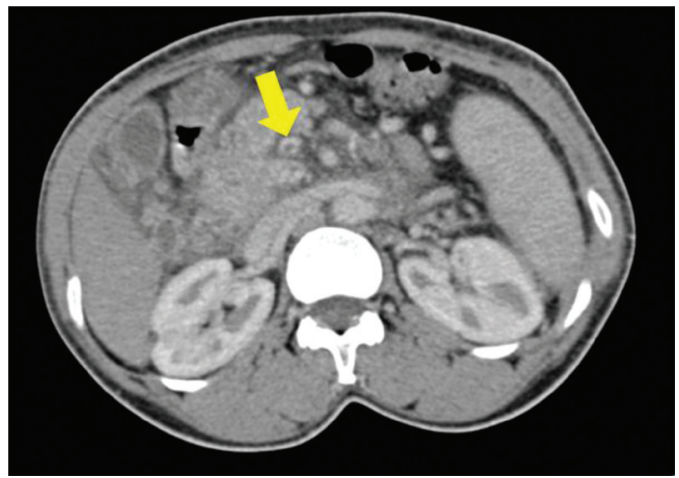

Fig. 6 Axial portal phase contrast computed tomography images show filling defect (arrow) in the superior mesenteric vein suggestive of thrombosis.

\section{Discussion}

"PCC" is defined as abnormalities in the extrahepatic biliary system including the gallbladder and cystic duct with or without abnormalities in the first and second generation biliary ducts in a patient with portal cavernoma. ${ }^{2}$ The two main causes of PCC include-extrahepatic portal vein occlusion and chronic portal vein thrombosis. In both conditions, the common precipitating factor is the presence of multiple periportal collaterals, which compress the bile duct and finally lead to stricture formation. ${ }^{3}$ There are two main venous channels around the CBD-“Petren's paracholedochal plexus," which lies around the wall (larger veins), and "Saint's epicholedochal plexus," which lies within the wall of the CBD (smaller veins) which are interconnected with transverse channels. Enlargement of the former is responsible for indentations in the bile duct walls, whereas enlargement of the latter causes enhancing bile duct wall thickening. ${ }^{3}$ Both the plexus of veins drain into 3 'o clock and 9'o clock marginal veins. Near the hilum these marginal veins enter into branches of portal vein. The marginal veins are connected to right gastric vein, cystic vein, left gastric vein, posterior superior pancreaticoduodenal vein, gastrocolic trunk, first jejunal vein, and occasionally to secondorder tributaries of superior mesenteric vein ( $\mathbf{F i g . ~ 9 )}{ }^{4,5}$ Walser et $\mathrm{al}^{3}{ }^{3}$ in their study on patients with chronic portal vein thrombosis with and without PCC, observed that there were a different set of collaterals in the two groups. Patients without PCC preferentially had gastroesophageal collaterals, and the cause was portal hypertension since the level of occlusion is usually at the first collateral, the coronary vein. Patients having PCC had portomesenteric occlusion with collaterals around the pancreatic head, and this was commonly seen in those with hypercoagulability as the cause for portal vein thrombosis. The first collaterals in these patients were usually from the gastrocolic trunk. ${ }^{3}$ The changes caused by PCC can be reversible or irreversible, and the reversibility depends on the etiology of such changes. Features like the bile duct's irregular walls, smooth strictures, and indentations are reversible with TIPS and are due to extrahepatic portal vein occlusion. On the other hand, rigid strictures, angulations, and the associated duct dilatation due to portal vein thrombosis affect the microvascular circulation or associated hepatic artery thrombosis, causing ischemia. These changes are considered irreversible. ${ }^{4}$ Also, an estimated 5 to $20 \%$ of patients develop choledocholithiasis, which can be isolated. Cholelithiasis can also occur, which is explained by stasis of bile due to strictures or narrowing, reduced contractility of the gallbladder due to collaterals in its wall, and portal vein thrombosis inducing atrophy of the liver, which in turn causes reduced flow of bile. ${ }^{3,4}$

PCC can occasionally present as a mass due to extensive wall thickening and connective tissue deposition around the bile ducts, as in our case. Kessler et al, ${ }^{6}$ in their case series of patients with PCC, reported two patients with the massforming type-one at the porta hepatis and one close to the head of the pancreas. The mass-like lesions were enhancing and were initially suspected of malignancy. But one pointer 

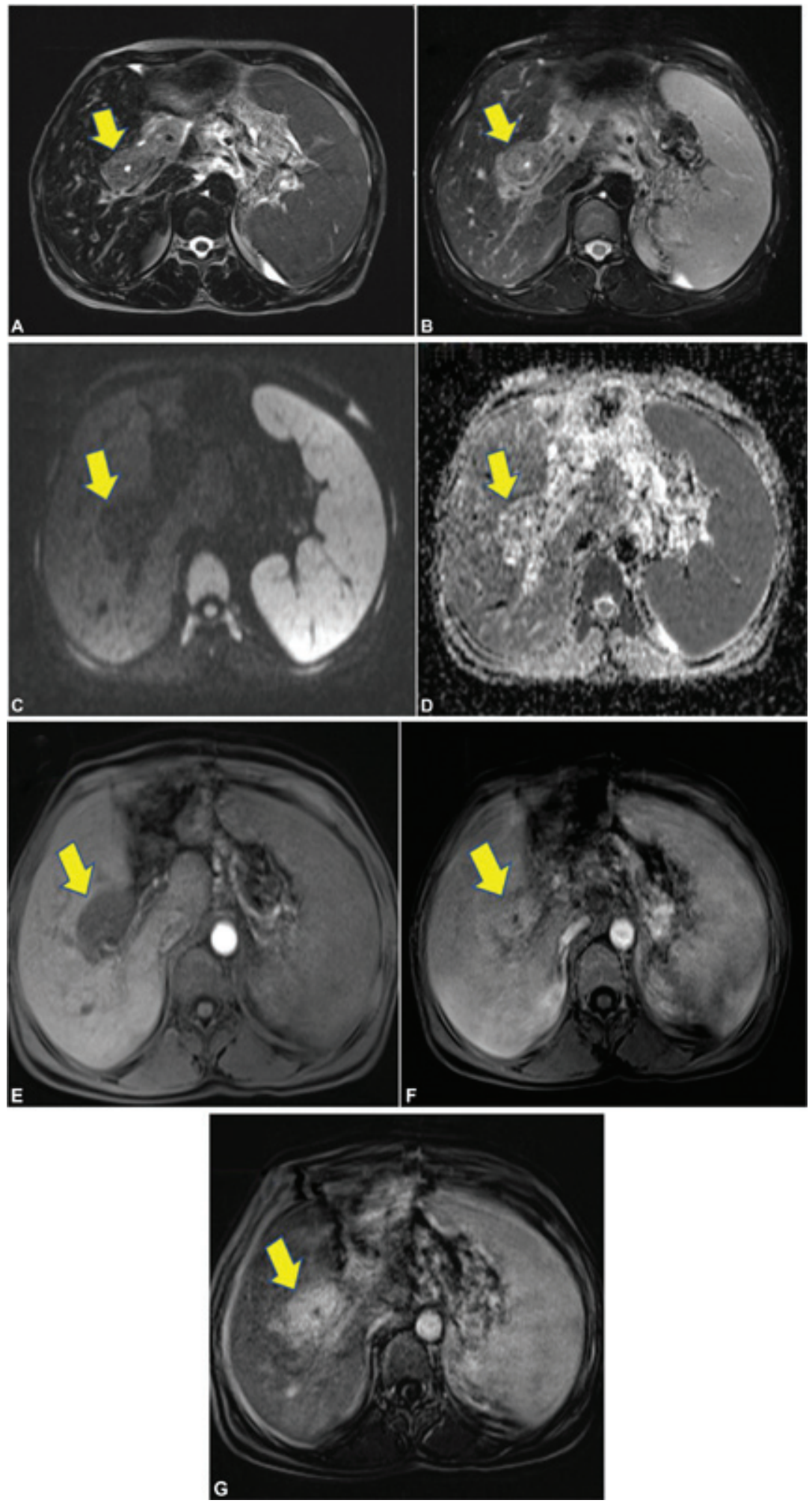

Fig. 7 Axial T2 (A), T2 fat suppressed (B) images show hyperintense wall thickening (arrows) involving the common bile duct (CBD) with no diffusion restriction (arrows) (C, D). Post gadolinium arterial (E), portal (F), and delayed phase subtracted (G) images show diffuse homogeneous persistent enhancement of the CBD wall thickening (arrows). 

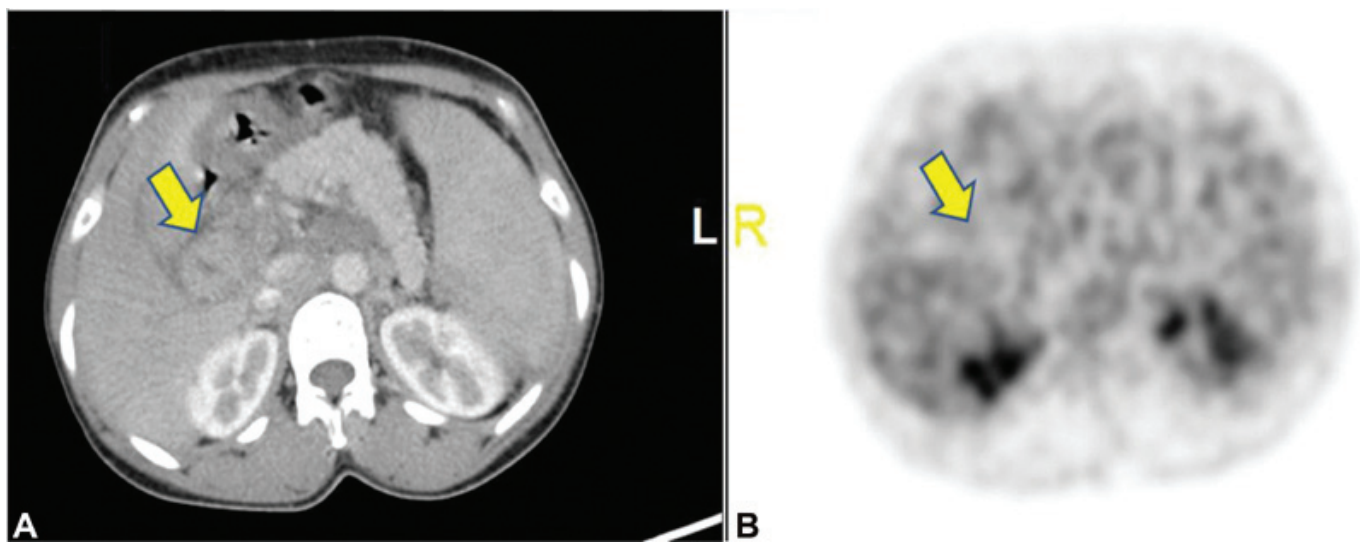

Fig. 8 (A, B) Positron emission tomography computed tomography (B) shows no obvious metabolic activity (arrows) in the concentric wall thickening of the common bile duct seen in the axial contrast-enhanced computed tomography image (A).
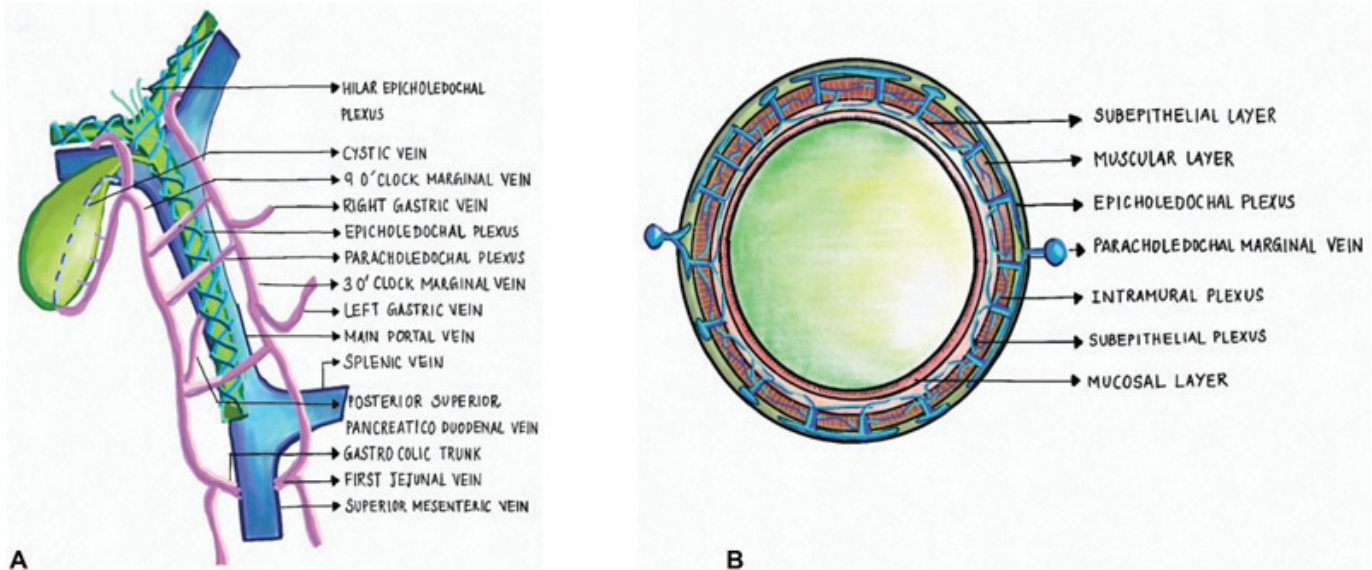

B

Fig. 9 (A, B) Coronal and cross-sectional schematic diagrams show paracholedochal and epicholedochal venous plexuses. The "Saint's epicholedochal venous plexus" drain into two marginal veins of the "Petren's paracholedochal venous plexus" at 3 o'clock and 9 o'clock positions which are interconnected with transverse channels. Both the plexus of veins drain into 3 o'clock and 9 o'clock marginal veins. Near the hilum these marginal veins enter into branches of portal vein. The marginal veins are connected to the right gastric vein, cystic vein, left gastric vein, posterior superior pancreaticoduodenal vein, gastrocolic trunk, first jejunal vein, and occasionally to second-order tributaries of superior mesenteric vein.

toward PCC was the presence of collateral vessels within the lesion, which could also be seen in our patient (-Fig. 5). ${ }^{6}$ Another case of mass-forming PCC has been reported by Hulshoff et al. ${ }^{7}$ One distinct finding in their case was homogeneous delayed phase enhancement without any enhancement in the arterial phase, which was attributed to increased deposition of connective tissue due to ischemia. ${ }^{7}$ Our case also had such homogeneous enhancement in the delayed phase (-Figs. 4 and 7 ).

Mass-forming PCC can confuse diseases like cholangiocarcinoma, primary CBD lymphoma, and IgG4 cholangiopathy. The irregular thickening of the CBD in PCC is referred to as the "pseudo-cholangiocarcinoma sign." Color Doppler can be a good differentiating tool as in PCC. The collaterals within the lesion show waveforms similar to that of the portal vein, whereas in cholangiocarcinoma, the tumoral vessels would show arterial waveforms. ${ }^{7}$ Primary lymphoma of the CBD is rare. It can be isolated or can occur as one of the many sites of systemic involvement of lymphoma. Compression of the CBD by lymph nodes is much more common than its wall's direct involvement. In primary lymphoma, despite diffuse wall thickening of the bile duct, there is only smooth and mild narrowing on cholangiography, without any mucosal irregularities. ${ }^{8,9}$ IgG4-related sclerosing cholangitis, also called autoimmune cholangiopathy, is a chronic inflammatory disease of the biliary system that manifests most commonly associated with other manifestations of IgG4-related systemic disease. However, it can also occur as an isolated pathology. MRCP findings include bile duct wall thickening, single or multifocal involvement, smooth margins of wall thickening, long segment stricture, the visible lumen in the thickened segments, and delayed homogeneous contrast enhancement. ${ }^{10}$ But in contrast to PCC, wall thickening in IgG4-related sclerosing cholangitis will show iso- to hypointense signal changes on T2weighted images with diffusion restriction. ${ }^{10}$ In our case, IgG4 levels were normal, and the wall thickening showed a hyperintense signal on T2-weighted images with no diffusion restriction. There was no CBD stricture. 
Our case presented with mass-like diffuse and vascular CBD wall thickening with patent lumen on ultrasound which led to further workup with CECT and MRI. The wall thickening showed persistent delayed enhancement, no significant diffusion restriction, and associated portal vein, superior mesenteric, and splenic vein thrombosis with multiple mesenteric collaterals. A PET-CT scan also ruled out malignant disease as there was no uptake. Thus, mass-forming PCC was diagnosed by exclusion. There were no significant obstructive biliopathy features in our patient which may be due to the earlier presentation. At present, there is no consensus on the optimal management of PCC as symptomatic PCC is rare and that it is difficult to access enough patients to allow meaningful comparison of different management options. Management of portal hypertension and relief of obstructive jaundice are the two main targets of PCC management. Recent international consensus papers on extrahepatic portal venous obstruction and portal hypertension have made the following statements regarding the management of PCC: (1) asymptomatic PCC does not require treatment; (2) endoscopic therapy is recommended in symptomatic PCC; (3) endoscopic sphincterotomy and stone extraction is recommended where CBD stones are present; (4) biliary stenting with or without dilatation is recommended in cases of bile duct stricture; (5) portosystemic shunt surgery should be considered wherever possible, particularly following failed biliary stenting, and (6) hepaticojejunostomy should be considered where other measures have failed. ${ }^{11}$ Our patient received conservative management and regular follow-up.

\section{Conclusion}

Patients with mass-forming PCC may be asymptomatic or mildly symptomatic, as in our case. Imaging findings can mimic primary CBD lymphoma, IgG4-related sclerosing cholangitis, and cholangiocarcinoma. Thus, we need to be aware of mass-forming PCC imaging findings to avoid unnecessary invasive interventions.

Conflict of Interest

None declared.

\section{References}

1 Fraser J, Brown AK. A clinical syndrome associated with a rare anomaly of the vena portal system. Surg Gynecol Obstet 1944; 78:520

2 Dhiman RK, Saraswat VA, Valla DC, et al. Portal cavernoma cholangiopathy: consensus statement of a working party of the Indian national association for study of the liver.J Clin Exp Hepatol 2014;4(Suppl 1):S2-S14

3 Walser EM, Runyan BR, Heckman MG, et al. Extrahepatic portal biliopathy: proposed etiology on the basis of anatomic and clinical features. Radiology 2011;258(01):146-153

4 Khuroo MS, Rather AA, Khuroo NS, Khuroo MS. Portal biliopathy. World J Gastroenterol 2016;22(35):7973-7982

5 Sharma M, Rameshbabu CS. Portal cavernoma cholangiopathy: an endoscopic ultrasound based imaging approach. J Clin Exp Hepatol 2014;4(Suppl 1):S53-S61

6 Kessler A, Graif M, Konikoff F, et al. Vascular and biliary abnormalities mimicking cholangiocarcinoma in patients with cavernous transformation of the portal vein: role of color Doppler sonography. J Ultrasound Med 2007;26(08):1089-1095

7 Hulshoff JB, Cuperus FJC, de Haas RJ. Mass-forming portal biliopathy presenting as extreme wall-thickening of the common bile duct. Diagnostics (Basel) 2020;10(09):623

8 Tartar VM, Balfe DM. Lymphoma in the wall of the bile ducts: radiologic imaging. Gastrointest Radiol 1990;15(01):53-57

9 Zakaria A, Al-Obeidi S, Daradkeh S. Primary non-Hodgkin's lymphoma of the common bile duct: a case report and literature review. Asian J Surg 2017;40(01):81-87

10 Madhusudhan KS, Das P, Gunjan D, Srivastava DN, Garg PK. IgG4related sclerosing cholangitis: a clinical and imaging review. AJR Am J Roentgenol 2019;213(06):1221-1231

11 Cellich PP, Crawford M, Kaffes AJ, Sandroussi C. Portal biliopathy: multidisciplinary management and outcomes of treatment. ANZ J Surg 2015;85(7-8):561-566 